\title{
Reduced Silent Occlusions with a Novel Catheter Infusion Set (BD FlowSmart): Results from Two Open-Label Comparative Studies
}

\author{
Michael Gibney, RN, MA, Zhenyi Xue, MS, ${ }^{2}$ Monica Swinney, PhD, ${ }^{3}$ \\ Damian Bialonczyk, PharmD, ${ }^{1,4}$ and Laurence Hirsch, MD ${ }^{1}$
}

\begin{abstract}
Background: Insulin pump users experience periods of unexplained hyperglycemia. In some cases these may be due to insulin flow interruptions termed "silent occlusions," which occur without activating the pump alarm and may require set replacement.

Materials and Methods: In-line pressure profiles of a novel infusion set with a 6-mm, 28-gauge polymer, dualported catheter (BD FlowSmart ${ }^{\mathrm{TM}}$; Becton Dickinson and Co., Franklin Lakes, NJ) were compared with those of an existing infusion set (Quick-set ${ }^{\circledR}$; Medtronic MiniMed, Northridge, CA) in two separate studies involving insulin diluent infusions over $2.5-4.5$-h periods in healthy adults without diabetes. Study 1 , a pilot study $(n=25)$, compared the occurrence of flow interruption events (silent occlusions and/or occlusion alarms) between the two infusion sets and between manual or device-assisted insertion methods. Study $2(n=60)$ was designed to show $\geq 50 \%$ reduction in flow interruption events with the BD set after manual insertions. (Silent occlusions were defined by a continuous pressure rise for $\geq 30 \mathrm{~min}$.)

Results: In Study 1, significantly fewer silent occlusions were seen with BD FlowSmart versus Quick-set infusion sets for both manual (three of 22 [13.6\%] vs. 12 of 24 [50\%]; $P=0.012$ ) and mechanical (two of 24 [8.3\%] vs. nine of 25 [36\%]; $P=0.037$ ) insertions, yielding risk reductions of $73 \%$ (95\% confidence interval [CI], 25-91\%) and 77\% (95\% CI, 17-94\%), respectively. In Study 2, flow interruption events occurred in three of $117(2.6 \%)$ and 12 of $118(10.2 \%)$ BD FlowSmart and Quick-set infusion sets, respectively, yielding a 75\% risk reduction $(95 \%$ CI, 20-92\%; $P=0.030)$. Percentage of time with flow interruption was significantly lower with BD sets in both studies $(P<0.02)$. Leakage $(>0.5 \mathrm{IU}$ or $5 \mu \mathrm{L})$ occurred infrequently and did not differ between sets.

Conclusions: A novel side-ported insulin infusion set demonstrated significant reductions in flow interruptions, including silent occlusions, versus a leading marketed set, which may improve insulin delivery.
\end{abstract}

\section{Introduction}

C ONTINUOUS SUBCUTANEOUS (SC) INSULIN INFUSION (CSII) using an insulin pump has been available as an insulin delivery method, primarily to people with type 1 dia- betes, for over 30 years. ${ }^{1}$ Since their introduction, technological advances in pumps have led to the availability of multiple basal rates, bolus wizards, different bolus modes, and, most recently, integration with continuous glucose monitoring. ${ }^{2,3}$ Although the latter has shown better glycemic control versus

\footnotetext{
${ }^{1}$ Diabetes Care, BD Medical Affairs, Becton Dickinson and Company, Franklin Lakes, New Jersey.

${ }^{2}$ Office of Science, Medicine and Technology, BD Corporate Clinical Development, Becton Dickinson and Company, Sparks, Maryland.

${ }^{3}$ Diabetes Care, BD Research \& Development, Becton Dickinson and Company, Andover, Massachusetts.

${ }^{4}$ School of Pharmacy, MCPHS University, Boston, Massachusetts.

These findings have been presented in part at the 2015 meetings of the Advanced Technologies \& Treatments for Diabetes, held in Paris, France, in February 2015, the American Diabetes Association, held in Boston, Massachusetts, in June 2015, and the European Association for the Study of Diabetes, held in Stockholm, Sweden, in September 2015.
}

(C) The Author(s) 2016; Published by Mary Ann Liebert, Inc. This Open Access article is distributed under the terms of the Creative Commons Attribution Noncommercial License (http://creativecommons.org/licenses/by-nc/4.0/) which permits any noncommercial use, distribution, and reproduction in any medium, provided the original author(s) and the source are credited. 
multiple daily injections plus self-monitoring of blood glucose, most of the improvement appears due to addition of continuous glucose monitoring, rather than CSII per se., However, the advances achieved with insulin pumps have overshadowed improvements with an insulin infusion set (IIS), which is required to deliver pumped insulin to the SC tissue.

The IIS is often considered to be the "Achilles heel" of CSII, and issues with the sets are one of the major reasons for patient frustrations with CSII, including discontinuing insulin pump therapy. ${ }^{6}$ These infusion set-related complications include insertion site, technical, and both metabolic and nonmetabolic manifestations. ${ }^{6,7}$ Recently, the American Diabetes Association and the European Association for the Study of Diabetes issued a joint statement on insulin pump benefits and risks, in which the paucity of publications related to IISs and insulin flow blockage was contrasted with the large number of IIS-related adverse events on the Food and Drug Administration MAUDE database. ${ }^{8}$ Additionally, reports of CSII delivery irregularities, where patients experience elevations in blood glucose levels that may or may not respond to a correction bolus (often called "unexplained hyperglycemia"), have been underappreciated. ${ }^{9}$

Infusion pumps are designed to alarm when an occlusion occurs (i.e., the in-line pressure reaches the pump's pressure alert threshold). van Bon et al..$^{9}$ reported that approximately $60 \%$ of patients with type 1 diabetes using CSII experienced at least one episode of unexplained hyperglycemia during a 13-week study period, and $30 \%$ of subjects experienced an occlusion alarm. Combined, about two-thirds of subjects in that study had one or more of these events. Unexplained and unresponsive hyperglycemia during CSII that occurs without triggering an occlusion alarm raises the concern that "silent" occlusions may be occurring.

Similar irregularities in fluid delivery during CSII using inline pressure as a surrogate indicator of insulin flow were initially observed in a randomized, open-label crossover pharmacokinetic study of intradermal infusion. ${ }^{10}$ Significant increases in pressure during the control, SC infusion periods (indicative of flow interruptions) were detected over a 24-h period in 20 adults with type 1 diabetes, many times without triggering the insulin pump occlusion alarm. Such interruptions may have clinical importance because it often takes several hours after an occlusion occurs before the pump occlusion alarm alerts the user, because of different thresholds for alarm activation and variation in basal infusion rate, length of the infusion catheter, and volume of bolus(es). ${ }^{11}$

These previously described limitations associated with conventional CSII led to the development of a novel CSII infusion set with a dual (end- and side)-ported catheter (BD FlowSmart ${ }^{\mathrm{TM}}$; Becton Dickinson and Co., Franklin Lakes, $\mathrm{NJ})$ designed to reduce flow interruptions and improve insulin delivery consistency. Preclinical studies indicate that the new infusion set reduces the frequency of flow interruptions, including silent occlusions. ${ }^{12,13}$ Here we report on two separate investigations comparing the effect of the BD FlowSmart set on insulin diluent delivery versus an existing IIS in healthy adults without diabetes.

\section{Research Design and Methods}

\section{Study objectives}

Study $1(n=25)$ and Study $2(n=60)$ were both open-label, randomized studies involving healthy subjects without dia- betes. Study 1, a pilot study, was intended to validate the performance of the investigational infusion set prior to the conduct of a larger, hypothesis-driven, comparative study (Study 2).

In Study 1, the primary objective was to evaluate the effect of the insertion method (manual vs. mechanically assisted) with the BD FlowSmart versus Quick-set ${ }^{\circledR}$ (Medtronic MiniMed, Northridge, CA) infusion sets on the occurrence of flow interruptions (silent occlusions). Secondary objectives included the occurrence of occlusion alarms during diluent delivery. The incidence of leakage from the insertion site upon set removal was also recorded.

In Study 2, the primary objective was to determine the rate of flow interruptions (silent occlusions and/or occlusion alarms) following manual insertion with the BD FlowSmart versus the Quick-set infusion sets. Secondary objectives included determination of the incidence and quantity of fluid leakage.

\section{Subjects}

Inclusion/exclusion criteria were similar for both studies. Subjects were eligible to participate if they were $18-75$ years of age, in generally good health, and with no acute or significant illness. Subjects were excluded if they were pregnant (self-reported), currently taking antiplatelet therapy or anticoagulants, had insulin-treated diabetes, a history of recurrent dermatological conditions, or gross skin abnormalities.

Both studies were completed at TKL Research, Inc. (Fairlawn, NJ) in July and August 2014. The protocols and supporting documents were reviewed and approved by the IntegReview Investigational Review Board, Austin, TX. All subjects provided written, informed consent. Study conduct was consistent with Good Clinical Practice standards and the Declaration of Helsinki.

\section{Materials}

The BD FlowSmart is an SC infusion set with a 6-mm, dual-ported, 28-gauge, polymer catheter and 30-gauge introducer needle (Fig. 1). It is inserted at a $90^{\circ}$ angle to the body and designed to be worn for up to 3 days with either luer-compatible or Medtronic Paradigm ${ }^{\circledR}$-compatible pumps/ reservoirs. The BD infusion set may be inserted manually or with the Medtronic insertion device. The Quick-set has a 6-mm 25-gauge cannula and 27-gauge introducer needle,

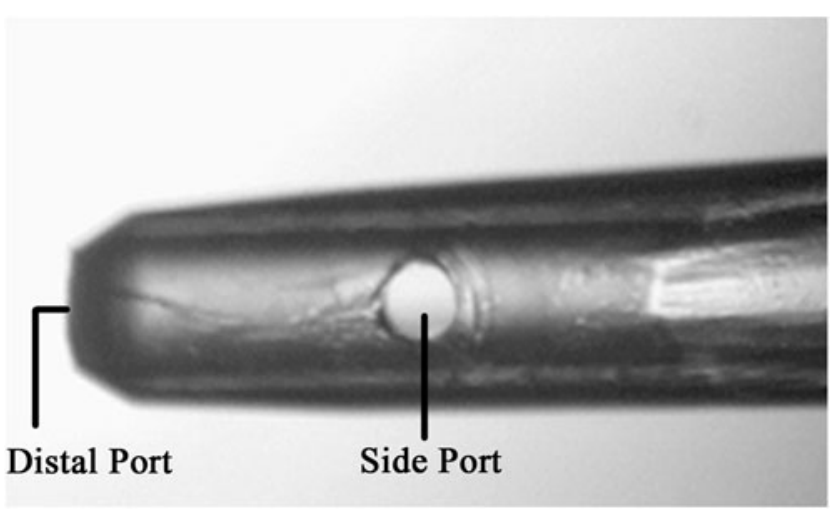

FIG. 1. BD FlowSmart dual-ported subcutaneous insulin infusion catheter tip. 
which may be inserted either manually or mechanically at a $90^{\circ}$ angle with the insertion device.

Humalog ${ }^{\circledR}$ (Lilly USA, LLC, Indianapolis, IN) insulin diluent $(1.6 \%$ glycerin, $0.16 \%$ metacresol, $0.065 \%$ phenol, and $0.38 \%$ dibasic sodium phosphate [wt/vol] in water) was used as a surrogate for insulin in both studies and was delivered using MiniMed ${ }^{\circledR}$ 530-gauge insulin infusion pumps (Medtronic MiniMed).

For measuring pressure, a DTX ${ }^{\mathrm{TM}}$ TNF-R sterile, singleuse, disposable pressure transducer (Argon Medical Devices Inc., Plano, TX) and either custom-made or commercially available microbore extension tubing were placed in-line between the pump and the infusion set (Fig. 2). Data from the pressure transducers were collected via a V-Link voltage/ strain gauge (LORD Microstrain, Williston, VT) with LabVIEW (National Instruments Corp., Austin) user interface.

\section{Methods}

In both studies, four infusion sets (two BD FlowSmart and two Quick-set) were inserted into randomly prespecified abdominal quadrants by trained nurses. In Study 1, one of each set was inserted manually, and the other was placed with a mechanical inserter to investigate the impact of insertion method on IIS performance; in Study 2, all sets were inserted manually. All pump reservoirs were filled with insulin diluent and connected to extension tubing; in-line pressure transducers (connected to the data logger) and infusion sets were primed before insertion (Fig. 2).

In both studies insulin diluent was delivered via insulin pump at a basal rate of $0.01 \mathrm{~mL} / \mathrm{h}$ (equivalent to $1.0 \mathrm{U} / \mathrm{h}$ of $\mathrm{U}-100$ insulin) for $2-3 \mathrm{~h}$. A bolus of $0.1 \mathrm{~mL}$ diluent (equivalent to $10 \mathrm{U}$ ) was then given. Basal conditions were continued, and tubing was clamped approximately $10 \mathrm{~min}$ or $1 \mathrm{~h}$ postbolus, as a positive control. While clamped, basal conditions were continued for a further $30 \mathrm{~min}$, and the infusion set was removed (Fig. 3).

In-line pressure was measured throughout the infusion, and pump occlusion alarms were recorded as they occurred. A silent occlusion was defined as a continuous pressure rise for $\geq 30$ min without an occlusion alarm (Fig. 4), although in some cases inline pressure may continue to rise and, over time, lead to an occlusion alarm. Upon set removal, the set and insertion site were examined for leakage, and, if applicable, fluid was swabbed and weighed (for quantification). A successful infusion was defined as measured leakage volume $\leq 5 \mu \mathrm{L}$ (equivalent to $\leq 0.5 \mathrm{U}$ ) of diluent on the skin/device interface.

\section{Sample size}

In the pilot study, a minimum of 20 insertions under each of the four conditions (BD FlowSmart or Quick-set, manual or mechanical insertion) was expected to provide sufficient information on the number of unexpected failures or differences between insertion methods; therefore 25 subjects were enrolled.

Subsequently, for Study 2, it was hypothesized that the BD FlowSmart would reduce flow interruptions (pressure rise events) by $\geq 50 \%$ compared with the Quick-set infusion set. Given the occurrence rate of such events observed with the Quick-set in Study 1 (approximately 35\% of sets), each infusion set type required 100 tests, giving a total of 200 insertions to maintain $80 \%$ statistical power in Study 2 (two-sided test with $\alpha=0.05$ ). Allowing for some unsuccessful infusions (e.g., catheter kinking or leakage of $>5 \mu \mathrm{L}$ ), a sample size of 60 subjects was specified in order to provide a total of 240 infusions, or 120 per set type.

\section{Statistical analysis}

Data from all successful infusions were used in the pressure profile analyses. Leakage occurrence/amounts and occlusion alarms were summarized by infusion set type with/without insertion device. Summary statistics were reported for pressure data. Silent occlusions and flow interruptions were calculated using an algorithm developed internally. Only continuous pressure rises lasting $\geq 30 \mathrm{~min}$ and that occurred prior to the intentional forced occlusions were included in calculations of silent occlusions. A flow interruption or pressure rise event was defined as occurrence of a silent occlusion, of a pump
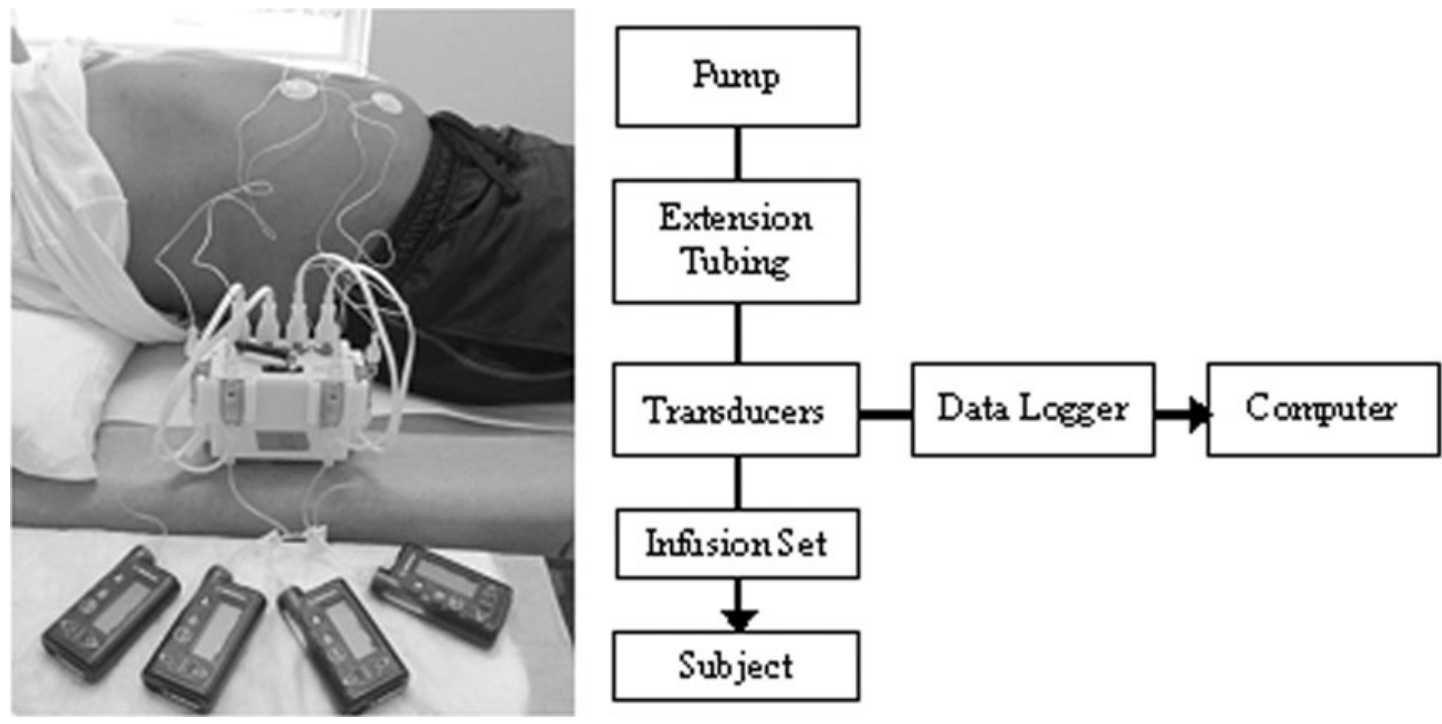

FIG. 2. Connection of infusion sets to in-line pressure transducers and the data logger. 


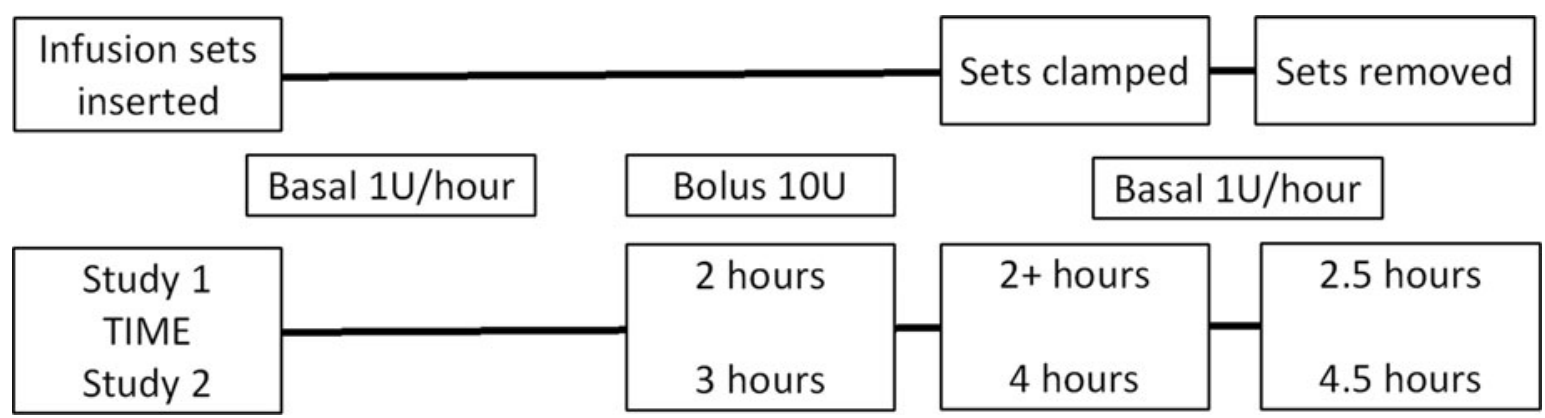

FIG. 3. Basal/bolus sequence and timing for Studies 1 and 2.

occlusion alarm, or of a silent occlusion that led to an occlusion alarm. Flow interruptions were reported as counts and percentages with $95 \%$ confidence intervals (CIs). A Fisher's exact test was used to compare the event rate between FlowSmart and Quick-set infusion sets. The proportion of time with flow interruption for each set type/insertion method was calculated as the total interruption time divided by total infusion time from start to clamp, and these values were compared by the Wilcoxon rank sum test. Data from all infusions were included in the summary of safety parameters.

\section{Results}

Of the 38 subjects screened for Study 1, 25 were enrolled, randomized, and completed the study. In Study 2, 75 subjects were screened; 63 met the inclusion/exclusion criteria, and three withdrew due to scheduling issues. The remaining 60 subjects were enrolled and completed the study.

\section{Demographics}

In total, 85 healthy subjects without diabetes participated in both studies. Subject demographics were similar between the two studies (Table 1).

\section{Silent occlusions}

In Study 1, the insertion method (mechanical versus manual) influenced the occurrence of silent occlusions. With the Medtronic set, silent occlusions occurred in more than one-third of the mechanically inserted and in one-half of the manually inserted sets (Table 2). With manual and mechan- ical inserter methods combined in Study 1, a total of five BD infusion sets experienced silent occlusions versus 21 with the Medtronic set; in Study 2 (all manual insertions) the numbers were one and 12, respectively (Table 3 ). In both studies, the new infusion set significantly reduced the risk of silent occlusions compared with the Medtronic set. In Study 1, significantly fewer silent occlusions were seen for both manually and mechanically inserted infusion sets, corresponding to significant risk reductions of $73 \%(95 \% \mathrm{CI}, 25-91 \% ; P=0.012)$ and $77 \%$ (95\% CI, 17-94\%; $P=0.037$ ), respectively. In Study 2, silent occlusions were significantly decreased by $92 \%$ with the new infusion set $(95 \%$ CI, 51-99\%; $P=0.003)$.

In Study 1, 95 infusions were included in the pressure analyses (five were removed because of measured leakage of $>5 \mu \mathrm{L}$ ). In the instances where flow interruptions were detected, the proportion of time with flow interruption was significantly lower with the BD FlowSmart than with the Quick-set with manual insertion $(P=0.006)$. Similar observations were made for infusions with mechanical insertions $(P=0.018)$ (Fig. 5).

In Study 2, 235 infusions were included in the pressure analysis (one was removed because of leakage and four for protocol deviations). One BD infusion set experienced a silent occlusion (with $16.4 \%$ time of flow interruption) versus 12 Quick-set infusion sets that had silent occlusions (median time with flow interruption, 14.9\%; $P=0.002$ ) (Fig. 5).

\section{Occlusion alarms}

In Study 1, there were in total seven occlusion alarms or no delivery alerts with the Medtronic set (five manual insertions

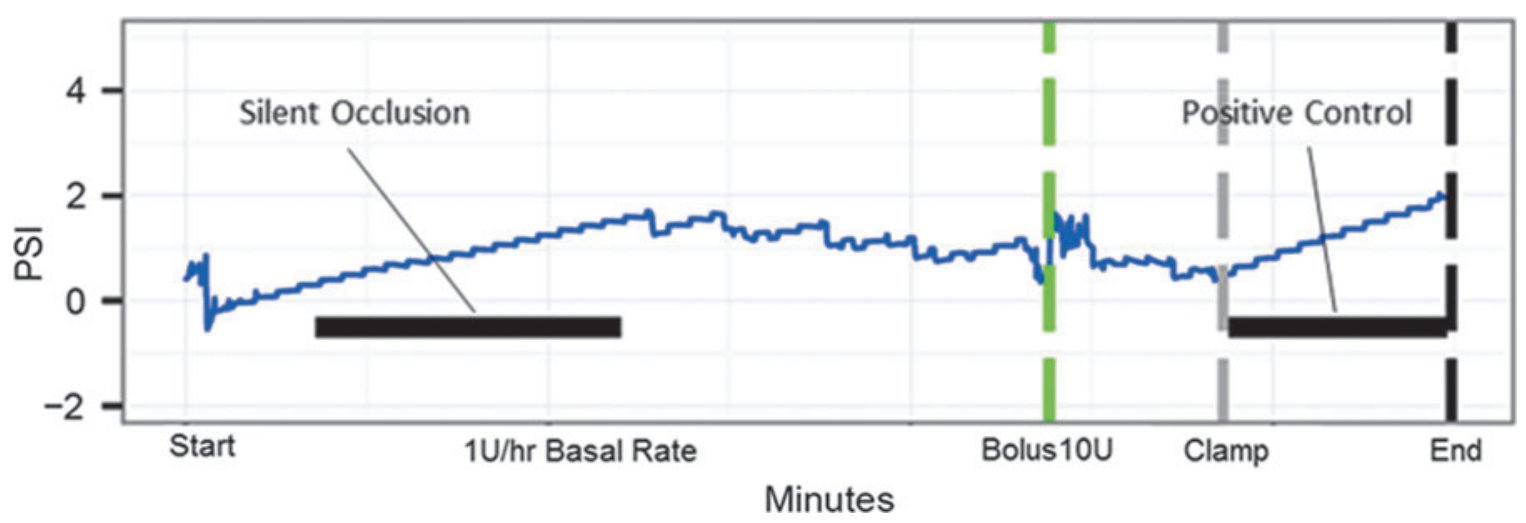

FIG. 4. Example of a pressure profile from Study 2 depicting a silent occlusion. PSI, pounds per square inch. 
Table 1. Baseline Demographics

\begin{tabular}{lcc}
\hline & Study $1(\mathrm{n}=25)$ & Study $2(\mathrm{n}=60)$ \\
\hline Female gender [n $(\%)]$ & $12(48 \%)$ & $27(45 \%)$ \\
Age (years) [mean & $39.1(14.9$, & $42.6(14.4$, \\
(SD, Min-Max)] & $19-62)$ & $18-75)$ \\
BMI (kg/m $\left.{ }^{2}\right)[$ mean & $28.7(5.8$, & $27.0(3.5$, \\
$\quad$ (SD, Min-Max)] & $20.2-46.6)$ & $21.1-35)$ \\
Race/ethnicity [n (\%)] & $10(40 \%)$ & $26(43.3 \%)$ \\
White/Caucasian & $7(28 \%)$ & $15(25 \%)$ \\
Black/African & & \\
American & $6(24 \%)$ & $14(23.3 \%)$ \\
Hispanic/Latino & $2(8 \%)$ & $4(6.7 \%)$ \\
Asian & $0(0 \%)$ & $1(1.7 \%)$ \\
Other &
\end{tabular}

BMI, body mass index; Max, maximum; Min, minimum.

and two with inserter) and none with the BD set (Table 2). There were no significant differences in occlusion alarms with either method of set insertion. Occlusion alarms were infrequent (total $n=3$ ) in Study 2, and no difference in such alarms was observed between the two sets in Study 2 (Table 3).

\section{Flow interruptions}

Combining silent occlusions and actual occlusion alarms, there were significantly fewer flow interruptions (pressure rise events) in Study 1 for both manual and device-assisted insertions. Relative risk reductions for the $\mathrm{BD}$ set versus the Medtronic set were 77\% (95\% CI, 38-92\%) and 79\% (95\% CI, 27-95\%), respectively. In Study 2, there were similar findings with all insertions done manually, yielding a significant $75 \%$ (95\% CI, 20-92\%) risk reduction in flow interruptions with the BD set.

\section{Leakage events}

Leakage events occurred infrequently; in both studies combined a total of six episodes of leakage occurred at the insertion site out of 340 sets $(1.8 \%)$. Four events were observed with the BD set in Study 1 (three with manual insertion and one with mechanical insertion) compared with one event with the Medtronic set (manual insertion). One event occurred with the BD set in Study $2(0.8 \%)$. There were no statistically significant differences in the occurrence of leakage between the study sets or with insertion methods.

\section{Safety}

In Study 1, one adverse event (mild erythema) was reported with the BD set, which was judged as most likely related to the study product adhesive. Fourteen episodes of mild erythema were reported in Study 2: seven each with the BD FlowSmart and Quick-set sets.

\section{Discussion}

Hyperglycemia is a well-described metabolic occurrence with insulin therapy, including treatment by CSII. ${ }^{11,14-18}$ With insulin pumps, hyperglycemia may result from infusion setrelated failure, specifically cannula withdrawal and occlusion or kinking of the catheter, which may reduce or block the flow of insulin from the pump to the SC tissue. ${ }^{7}$ Furthermore, effective insulin delivery with infusion sets also relies on prevention of fluid leakage at the insertion site (or at the reservoirtubing connection). If any of these is disrupted then the desired dose of insulin will not be delivered to the SC tissue.

It has been previously reported that blood glucose values rise $1 \mathrm{mg} / \mathrm{dL}$ for each minute an IIS is disconnected from the pump (for at least $30 \mathrm{~min}$ ), under basal infusion conditions. The elevation of blood glucose concentration persisted for $3-3.5 \mathrm{~h}$ after reconnecting the infusion set. ${ }^{19}$ These findings helped inform our definition of a silent occlusion as a continuous rise in in-line pressure for at least $30 \mathrm{~min}$. Furthermore, a $15 \%$ failure rate of polymer catheters (blood glucose value of $>250 \mathrm{mg} / \mathrm{dL}$ and not decreasing at least $50 \mathrm{mg}$ in $1 \mathrm{~h}$ post-correction bolus) over the 8-h period following insertion was observed by Patel et al. ${ }^{15}$ and in nearly $9 \%$ of soft cannula insertions within a 6-h period by Renard et al ${ }^{16}$ suggesting that existing polymer catheters have a tendency to fail upon insertion.

Reflecting these issues, insulin pumps are designed to alert patients when occlusion of the infusion set has occurred, by triggering an alarm when the in-line pressure reaches the pump's pressure alert threshold. These pressure thresholds

Table 2. Occurrence of Silent Occlusions, Percentage of Time with Flow Interruption, Occlusion Alarms, and Flow Interruptions Following Manual and Mechanical Insertion of the BD FlowSmart or Quick-SeT Infusion Set in Healthy Subjects Without Diabetes in Study 1

\begin{tabular}{|c|c|c|c|c|}
\hline $\begin{array}{l}\text { Infusion set, } \\
\text { insertion method }\end{array}$ & Silent occlusion ${ }^{\mathrm{a}, \mathrm{b}}$ & $\begin{array}{c}\% \text { of time with } \\
\text { flow interruption }^{\mathrm{c}, \mathrm{d}}\end{array}$ & Occlusion alarm ${ }^{\mathrm{b}}$ & Flow interruption $^{\mathrm{b}, \mathrm{e}}$ \\
\hline \multicolumn{5}{|l|}{ Quick-set } \\
\hline Inserter $(n=25)$ & $9(36.0 \%, 20.3-55.5 \%)$ & $26.7 \%(24.8-40.7 \%)$ & $2(8 \%, 2.2-25.0 \%)$ & $10(40 \%, 23.4-59.3 \%)$ \\
\hline Manual $(n=24)$ & $12(50.0 \%, 31.4-68.6 \%)$ & $34.9 \%(28.7-45.6 \%)$ & $5(20.8 \%, 9.2-40.5 \%)$ & $14(58.3 \%, 38.8-75.5 \%)$ \\
\hline \multicolumn{5}{|l|}{ FlowSmart } \\
\hline Inserter $(n=24)$ & $2(8.3 \%, 2.3-25.9 \%)^{\mathrm{f}}$ & $23.4 \%(22.3-24.5 \%)$ & $0(0 \%, 0-13.8 \%)$ & $2(8.3 \%, 2.3-25.9 \%)^{\mathrm{g}}$. \\
\hline Manual $(n=22)$ & $3(13.6 \%, 4.8-33.3 \%)^{\mathrm{h}}$ & $21.1 \%(19.8-39.5 \%)$ & $0(0 \%, 0-14.9 \%)^{\mathrm{i}}$ & $3(13.6 \%, 4.8-33.3 \%)^{j}$ \\
\hline \multirow{2}{*}{\multicolumn{5}{|c|}{ 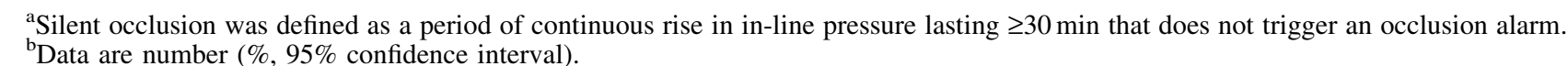 }} \\
\hline & & & & \\
\hline \multicolumn{5}{|c|}{${ }^{c}$ Data are median (interquartile range) values. } \\
\hline \multicolumn{5}{|c|}{${ }^{\mathrm{d}}$ Calculated as the total interruption time divided by total eligible infusion time from start to clamp. } \\
\hline \multicolumn{5}{|c|}{ All $P$ values related to comparisons between FlowSmart and Quick-set with associated insertion method: ${ }^{\mathrm{f}} P=0.037,{ }^{\mathrm{g}} P=0.018$, } \\
\hline
\end{tabular}


Table 3. Occurrence of Silent Occlusions, Percentage of Time with Flow Interruption, OcClusion Alarms, and Flow Interruptions Following InSERTion of a BD FlowSmart or Quick-SET Infusion Set in Healthy Subjects Without Diabetes in Study 2

\begin{tabular}{lcccc}
\hline Infusion set & Silent occlusion $^{\mathrm{a}, \mathrm{b}}$ & $\begin{array}{c}\text { \% of time with } \\
\text { flow interruption }^{\mathrm{c}, \mathrm{d}}\end{array}$ & ${\text { Occlusion } \text { alarm }^{\mathrm{b}}}^{\mathrm{O}}$ & Flow interruption $^{\mathrm{b}, \mathrm{e}}$ \\
\hline Quick-set $(n=118)$ & $12(10.2 \%, 5.9-16.9 \%)$ & $14.9 \%(11.9-24.6 \%)$ & $1(0.8 \%, 0.2-4.6 \%)$ & $12(10.2 \%, 5.9-16.9 \%)$ \\
FlowSmart $(n=117)$ & $1(0.9 \%, 0.2-4.7 \%)^{\mathrm{f}}$ & NA & $2(1.7 \%, 0.5-6.0 \%)$ & $3(2.6 \%, 0.9-7.3 \%)^{\mathrm{g}}$ \\
\hline
\end{tabular}

All infusion set insertions were done manually.

${ }^{a}$ Defined as a period of continuous rise in in-line pressure lasting $\geq 30 \mathrm{~min}$ that does not trigger an occlusion alarm.

${ }^{\mathrm{b}}$ Data are number (\%, 95\% confidence interval).

${ }^{\mathrm{c}}$ Data are median (interquartile range) values.

${ }^{\mathrm{d}}$ Calculated as the total interruption time divided by total eligible infusion time from start to clamp.

${ }^{\mathrm{e}}$ Flow interruption was defined as a silent occlusion and/or occlusion alarm.

${ }^{\mathrm{f}} P=0.003,{ }^{\mathrm{g}} P=0.030$ versus Quick-set.

NA, not available.

vary among pumps, such that rises in blood glucose concentration may last up to $5 \mathrm{~h}$ before the occlusion alarm sounds - depending on the pump, the alarm pressure setting, the type and length of infusion set tubing, etc. ${ }^{11}$ Without continuous glucose monitoring, these events are, by definition, silent. In addition, the same study demonstrated that the majority of in-line pressure increases during CSII occurring in a 24-h period did not trigger the pump alarm. ${ }^{11}$ These are silent occlusions - the clinical counterpart of the events described in the two studies reported here.

We believe our studies evaluate silent occlusion and flow interruption frequency and duration in a controlled manner with a new IIS for the first time in a clinical research setting. The data demonstrate that silent occlusions occur frequently ( $>35 \%$ of Quick-set infusion sets in Study 1) and were significantly reduced more than $75 \%$ with the BD FlowSmart infusion set. Additionally, similar reductions were determined for the proportion of time with occlusion.

These findings also confirm that silent occlusions can occur shortly after the insertion of the infusion set $(<4 \mathrm{~h}$ postinsertion); possible causes of these events may include catheter kinking, accidental insertion of the catheter into the muscle tissue, catheter obstruction due to tissue matter, obstruction due to fibrin formation (which might be expected to occur after longer infusion duration), insulin precipitation or aggregation, or a combination of these factors. Whatever the cause, pressure increased in-line, corresponding to a flow reduction.

A reduction in the number of occlusion alarms with the $\mathrm{BD}$ FlowSmart when compared with the Quick-set infusion set was observed in Study 1 but not Study 2. This is potentially due to improvements in insertion technique by study staff over the two study periods. Each infusion set was inserted by a trained clinician to standardize insertion in subjects without diabetes who were unfamiliar with insulin injections and CSII. This methodology has also been used in other studies involving IISs. ${ }^{15}$ The impact of self-insertion on the number of pressure-related events requires further investigation in patients using CSII.

Insulin leakage at the insertion site (or between pump reservoir and tubing connector) can also be a serious problem with CSII; a leaking infusion set may not be identified until
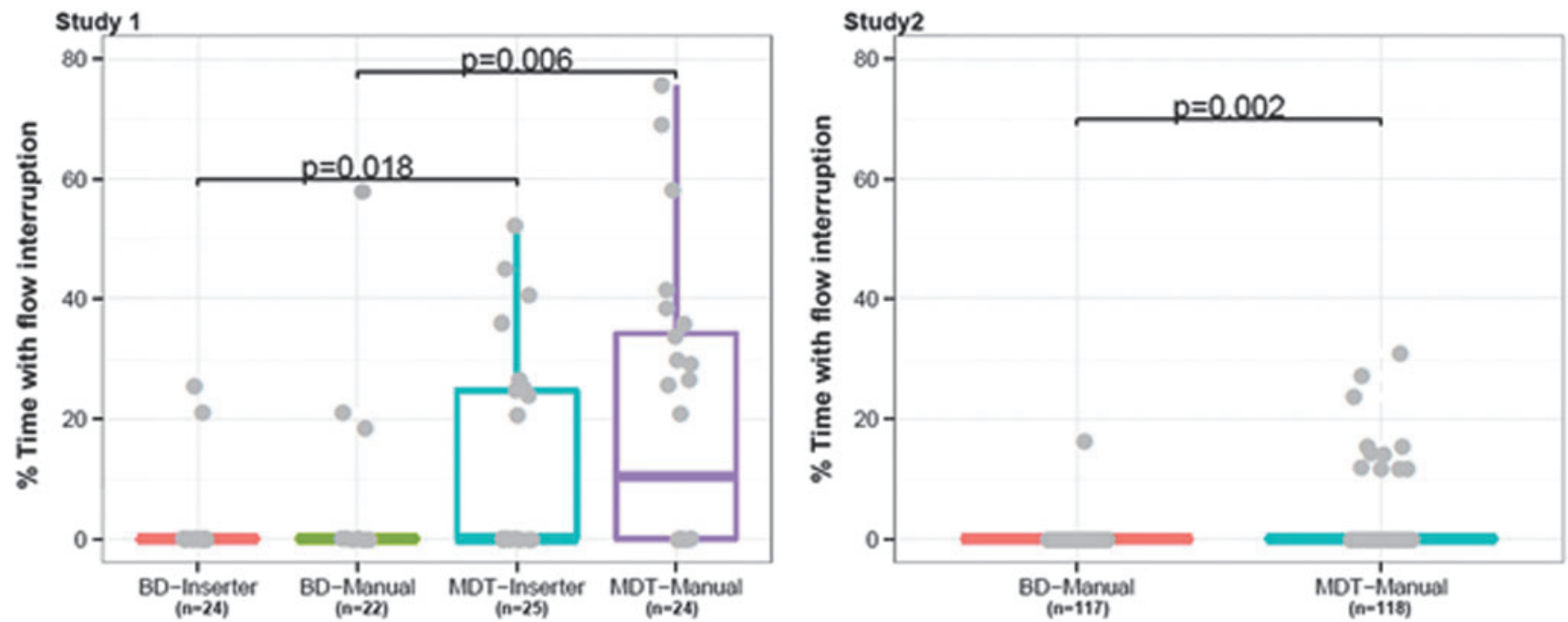

FIG. 5. Box plot of proportion of infusion time with flow interruption in both Studies 1 and 2. FlowSmart (BD) has significantly reduced the percentage of time with flow interruptions in Study 1 (manual and device-assisted insertions) and in Study 2 (manual insertions). Analysis used Wilcoxon rank sum testing. MDT, Medtronic MinMed Quick-set. 
the patient detects fluid under the set's adhesive pad or, more commonly, smells creosol or a similar phenolic preservative. ${ }^{17}$ In this scenario, the patient may also experience unexplained hyperglycemia. However, there are no pump alarms for leakage, leaving occurrence of hyperglycemia as the primary signal to the user. During these short studies, leakage occurred infrequently with both infusion sets.

A major limitation in these studies was the use of insulin diluent in healthy subjects as opposed to insulin in CSII patients. We do not believe this affects our findings, and it also minimized any potential patient risks at an early stage of product development. Similar changes in in-line pressure have been observed with insulin as with diluent in preclinical studies in swine with the BD FlowSmart set (N. Bolick, BD Technologies, personal communication, October 29, 2015). Other limitations, including the use of trained nurses to manage insertions rather than the subject, relatively short duration of infusions, and limited ambulatory activities by subjects, might not reflect the true prevalence of pressure-related or leakage events and unexplained hyperglycemia as reported by others ${ }^{9,16}$ but are inherent to the nature of the investigations performed. A controlled, multicenter study of longer duration in CSII patients with type 1 diabetes is needed to determine the relevance of these findings in real-world conditions. All authors are BD employees, but the measurements and findings were not influenced or biased by this factor.

Based on our findings as well as previous reports, ${ }^{15,16,18}$ we believe that silent occlusions contribute to the occurrence of unexplained hyperglycemia and glycemic variability in patients using CSII. Silent occlusions should be considered with any insulin pump patient who experiences unexplained hyperglycemia, especially in those whose glycemic excursion does not respond to a correction bolus and where the occlusion alarm has not sounded.

The infusion set with BD FlowSmart technology shares many characteristics with other commercially available infusion sets, including the insertion angle, composition of materials, and length of cannula. There are, however, modifications with the new set that have not been used previously in SC infusion sets. Most notable is the novel side-ported catheter, designed to improve insulin flow. Additionally, the new infusion set has a smaller gauge introducer needle and cannula, and the introducer needle is retained after its withdrawal. These modifications might provide reduced insertion pain and an improved user experience. Clearly, additional studies are needed to evaluate patient-reported outcomes with the new BD infusion set. Other questions of interest include potential effects on duration of set wear, as well as insulin pharmacokinetics and pharmacodynamics.

In conclusion, the new BD infusion set significantly reduced the occurrence of silent occlusions, flow interruptions, and time with flow interruption, without an increase in leakage at the insertion site, compared with a leading commercially available infusion set. Reduced flow interruptions may result in more consistent and reliable infusion of insulin and may help reduce the occurrence of hyperglycemic events and of glycemic variability in CSII users.

\section{Acknowledgments}

The authors wish to thank the JDRF and the Helmsley Charitable Trust for supporting and co-funding the development of the FlowSmart insulin infusion set. In addition, the authors wish to acknowledge the managers and staff at TKL Research, Inc., Fairlawn, NJ, who helped conduct the studies. In addition, we want to specifically acknowledge and thank Eric Bene, Rachael Turner, Diane Sutter, Jane Lawrence, and Natalie Bascom who helped coordinate and monitor study procedures or to complete data collection. We thank Ms. Jane Caple of Athena Education Group, LLC, paid by $\mathrm{BD}$, for assistance in revision, editing, and formatting of the manuscript.

\section{Author Disclosure Statement}

All authors are employees of BD.

All authors had complete access to the data and approved the decision to submit the manuscript for publication. M.G. and D.B. provided the initial draft. M.G. helped design the studies. Z.X. performed the statistical analyses. M.S. helped prepare the infusion set and pressure measuring equipment and monitored it during the studies. L.H. provided medical input, oversaw the compilation and interpretation of the data, and provided revisions of the article for content.

\section{References}

1. Pickup JC, Keen H, Parsons JA, et al.: Continuous subcutaneous insulin infusion: an approach to achieving normoglycaemia. BMJ 1978;1:204-207.

2. Weissberg-Benchell J, Antisdel-Lomaglio J, Seshadri R: Insulin pump therapy: a meta-analysis. Diabetes Care 2003; 26:1079-1087.

3. Heinemann L: Insulin pump therapy: what is the evidence for using different types of boluses for coverage of prandial insulin requirements? J Diabetes Sci Technol 2009;3:14901500 .

4. Bergenstal RM, Tamborlane WV, Ahmann A, et al.: Effectiveness of sensor-augmented insulin-pump therapy in type 1 diabetes. N Engl J Med 2010;363:311-320.

5. Garg SK, Voelmle MK, Beatson CR, et al.: Use of continuous glucose monitoring in subjects with type 1 diabetes on multiple daily injections versus continuous subcutaneous insulin infusion therapy. Diabetes Care 2011;34:574-579.

6. Heinemann L, Krinelke L: Insulin infusion set: the Achilles heel of continuous subcutaneous insulin infusion. J Diabetes Sci Technol 2012;6:954-964.

7. Pickup JC, Yemane, N, Brackenridge, A, et al.: Nonmetabolic complications of continuous subcutaneous insulin infusion: a patient survey. Diabetes Technol Ther 2014;16: 145-149.

8. Heinemann L, Flemming GA, Petrie JR, et al.: Insulin pump risks and benefits: a clinical appraisal of pump safety standards, adverse event reporting, and research needs. A Joint Statement of the European Association for the Study of Diabetes and the American Diabetes Association Technology Working Group. Diabetes Care 2015; 38:716-722.

9. van Bon AC, Bode BW, Sert-Langeron C, et al.: Insulin glulisine compared to insulin aspart and to insulin lispro administered by continuous subcutaneous insulin infusion in patients with type 1 diabetes: a randomized controlled trial. Diabetes Technol Ther 2011;13:607-614.

10. Keith S, McVey E, Pettis RJ: High sensitivity occlusion detection using fluid pressure monitoring during basal insulin infusion. Diabetes 2013;62(suppl 1):A249A250. 
11. van Bon AC, Dragt D, deVries JH: Significant time until catheter occlusion alerts in currently marketed insulin pumps at two basal rates. Diabetes Technol Ther 2012;14: 447-448.

12. Bolick N, Morel D, Gerth S, et al.: Reduction of silent occlusion occurrence during continuous subcutaneous insulin infusion [abstract]. Diabetes Technol Ther 2015;17(Suppl 1): A35.

13. Bolick N, Morel D, Alarcon J, et al.: In vivo flow evaluation and correlation to insulin pharmacokinetics of continuous subcutaneous insulin infusion sets [abstract]. Diabetes 2015;64(Suppl 1):A274-A275.

14. Cope JU, Samuels-Reid JH, Morrison AE: Pediatric use of insulin pump technology: a retrospective study of adverse events in children ages 1-12 years. J Diabetes Sci Technol 2012;6:1053-1059.

15. Patel PJ, Benasi K, Ferrari, G, et al.: Randomized trial of infusion set function: steel versus Teflon. Diabetes Technol Ther 2014;16:15-19.

16. Renard E, Guerci B, Leguerrier AM, et al.: Lower rate of initial failures and reduced occurrence of adverse events with a new catheter model for continuous subcutaneous insulin infusion: prospective, two-period, observational, multicenter study. Diabetes Technol Ther 2010;12:769-773.

17. Ponder SW, Skyler JS, Kruger DF, et al.: Unexplained hyperglycemia in continuous subcutaneous insulin infusion: evaluation and treatment. Diabetes Educ 2008;34:327-333.

18. Thethi TK, Rao A, Kawji H, et al.: Consequences of delayed pump infusion line change in patients with type 1 diabetes mellitus treated with continuous subcutaneous insulin infusion. J Diabetes Complications 2010;24:73-78.

19. Zisser H: Quantifying the impact of a short-interval interruption of insulin-pump infusion sets on glycemic excursions. Diabetes Care 2008;31:238-239.

Address correspondence to: Laurence Hirsch, MD Diabetes Care BD Medical Affairs Becton Dickinson and Company 1 Becton Drive Franklin Lakes, NJ 07417

E-mail: Laurence_hirsch@bd.com 